\title{
Research Article \\ Stability of a Quadratic Functional Equation in the Spaces of Generalized Functions
}

\author{
Young-Su Lee \\ Department of Mathematical Sciences, Korea Advanced Institute of Science and Technology, \\ 373-1 Guseong-dong, Yuseong-gu, Daejeon 305-701, South Korea \\ Correspondence should be addressed to Young-Su Lee, masuri@kaist.ac.kr \\ Received 30 June 2008; Accepted 20 August 2008 \\ Recommended by László Losonczi
}

Making use of the pullbacks, we reformulate the following quadratic functional equation: $f(x+y+$ $z)+f(x)+f(y)+f(z)=f(x+y)+f(y+z)+f(z+x)$ in the spaces of generalized functions. Also, using the fundamental solution of the heat equation, we obtain the general solution and prove the Hyers-Ulam stability of this equation in the spaces of generalized functions such as tempered distributions and Fourier hyperfunctions.

Copyright (C) 2008 Young-Su Lee. This is an open access article distributed under the Creative Commons Attribution License, which permits unrestricted use, distribution, and reproduction in any medium, provided the original work is properly cited.

\section{Introduction}

Functional equations can be solved by reducing them to differential equations. In this case, we need to assume differentiability up to a certain order of the unknown functions, which is not required in direct methods. From this point of view, there have been several works dealing with functional equations based on distribution theory. In the space of distributions, one can differentiate freely the underlying unknown functions. This can avoid the question of regularity. Actually using distributional operators, it was shown that some functional equations in distributions reduce to the classical ones when the solutions are locally integrable functions [1-4].

Another approach to distributional analogue for functional equations is via the use of the regularization of distributions [5,6]. More exactly, this method gives essentially the same formulation as in [1-4], but it can be applied to the Hyers-Ulam stability [7-10] for functional equations in distributions [11-14].

In accordance with the notions in [11-14], we reformulate the following quadratic functional equation:

$$
f(x+y+z)+f(x)+f(y)+f(z)=f(x+y)+f(y+z)+f(z+x)
$$


in the spaces of generalized functions. Also, we obtain the general solution and prove the Hyers-Ulam stability of (1.1) in the spaces of generalized functions such as $S^{\prime}\left(\mathbb{R}^{n}\right)$ of tempered distributions and $\mathcal{F}^{\prime}\left(\mathbb{R}^{n}\right)$ of Fourier hyperfunctions.

The functional equation (1.1) was first solved by Kannappan [15]. In fact, he proved that a function on a real vector space is a solution of (1.1) if and only if there exist a symmetric biadditive function $B$ and an additive function $A$ such that $f(x)=B(x, x)+A(x)$. In addition, Jung [16] investigated Hyers-Ulam stability of (1.1) on restricted domains, and applied the result to the study of an interesting asymptotic behavior of the quadratic functions.

As a matter of fact, we reformulate (1.1) and related inequality in the spaces of generalized functions as follows. For $u \in \mathcal{S}^{\prime}\left(\mathbb{R}^{n}\right)$ or $u \in \mathcal{F}^{\prime}\left(\mathbb{R}^{n}\right)$,

$$
\begin{gathered}
u \circ A+u \circ P_{1}+u \circ P_{2}+u \circ P_{3}=u \circ B_{1}+u \circ B_{2}+u \circ B_{3}, \\
\left\|u \circ A+u \circ P_{1}+u \circ P_{2}+u \circ P_{3}-u \circ B_{1}-u \circ B_{2}-u \circ B_{3}\right\| \leq \epsilon,
\end{gathered}
$$

where $A, B_{1}, B_{2}, B_{3}, P_{1}, P_{2}$, and $P_{3}$ are the functions defined by

$$
\begin{array}{cc}
A(x, y, z)=x+y+z, \\
P_{1}(x, y, z)=x, \quad P_{2}(x, y, z)=y, \quad P_{3}(x, y, z)=z, \\
B_{1}(x, y, z)=x+y, \quad B_{2}(x, y, z)=y+z, \quad B_{3}(x, y, z)=z+x .
\end{array}
$$

Here, $\circ$ denotes the pullbacks of generalized functions, and $\|v\| \leq \epsilon$ in (1.3) means that $|\langle v, \varphi\rangle| \leq \epsilon\|\varphi\|_{L^{1}}$ for all test functions $\varphi$.

As a consequence, we prove that every solution $u$ of inequality (1.3) can be written uniquely in the form

$$
u(x)=u\left(x_{1}, \ldots, x_{n}\right)=\sum_{1 \leq i \leq j \leq n} a_{i j} x_{i} x_{j}+\sum_{1 \leq i \leq n} b_{i} x_{i}+\mu,
$$

where $\mu$ is a bounded measurable function such that $\|\mu\|_{L^{\infty}} \leq 13 / 3$.

\section{Preliminaries}

We first introduce briefly spaces of some generalized functions such as tempered distributions and Fourier hyperfunctions. Here, we use the multi-index notations, $|\alpha|=\alpha_{1}+\cdots+\alpha_{n}$, $\alpha !=\alpha_{1} ! \cdots \alpha_{n} !, x^{\alpha}=x_{1}^{\alpha_{1}} \cdots x_{n}^{\alpha_{n}}$, and $\partial^{\alpha}=\partial_{1}^{\alpha_{1}} \cdots \partial_{n}^{\alpha_{n}}$, for $x=\left(x_{1}, \ldots, x_{n}\right) \in \mathbb{R}^{n}$ and $\alpha=\left(\alpha_{1}, \ldots, \alpha_{n}\right) \in \mathbb{N}_{0}^{n}$, where $\mathbb{N}_{0}$ is the set of nonnegative integers and $\partial_{j}=\partial / \partial x_{j}$.

Definition 2.1 (see $[17,18]$ ). One denotes by $\mathcal{S}\left(\mathbb{R}^{n}\right)$ the Schwartz space of all infinitely differentiable functions $\varphi$ in $\mathbb{R}^{n}$ satisfying

$$
\|\varphi\|_{\alpha, \beta}=\sup _{x \in \mathbb{R}^{n}}\left|x^{\alpha} \partial^{\beta} \varphi(x)\right|<\infty
$$


for all $\alpha, \beta \in \mathbb{N}_{0}^{n}$, equipped with the topology defined by the seminorms $\|\cdot\|_{\alpha, \beta}$. A linear functional $u$ on $\mathcal{S}\left(\mathbb{R}^{n}\right)$ is said to be tempered distribution if there are a constant $C \geq 0$ and a nonnegative integer $N$ such that

$$
|\langle u, \varphi\rangle| \leq C \sum_{|\alpha|,|\beta| \leq N} \sup _{x \in \mathbb{R}^{n}}\left|x^{\alpha} \partial^{\beta} \varphi\right|
$$

for all $\varphi \in \mathcal{S}\left(\mathbb{R}^{n}\right)$. The set of all tempered distributions is denoted by $\mathcal{S}^{\prime}\left(\mathbb{R}^{n}\right)$.

Imposing the growth condition on $\|\cdot\|_{\alpha, \beta}$ in (2.1), a new space of test functions has emerged as follows.

Definition 2.2 (see [19]). One denotes by $\mathcal{F}\left(\mathbb{R}^{n}\right)$ the Sato space of all infinitely differentiable functions $\varphi$ in $\mathbb{R}^{n}$ such that

$$
\|\varphi\|_{A, B}=\sup _{x, \alpha, \beta} \frac{\left|x^{\alpha} \partial^{\beta} \varphi(x)\right|}{A^{|\alpha|} B^{|\beta|} \alpha ! \beta !}<\infty
$$

for some positive constants $A, B$ depending only on $\varphi$. One says that $\varphi_{j} \rightarrow 0$ as $j \rightarrow \infty$ if $\left\|\varphi_{j}\right\|_{A, B} \rightarrow 0$ as $j \rightarrow \infty$ for some $A, B>0$, and denotes by $\mathcal{F}^{\prime}\left(\mathbb{R}^{n}\right)$ the strong dual of $\mathcal{F}\left(\mathbb{R}^{n}\right)$ and calls its elements Fourier hyperfunctions .

It can be verified that the seminorms (2.3) are equivalent to

$$
\|\varphi\|_{h, k}=\sup _{x \in \mathbb{R}^{n}, \alpha \in \mathbb{N}_{0}^{n}} \frac{\left|\partial^{\alpha} \varphi(x)\right| \exp k|x|}{h^{|\alpha|} \alpha !}<\infty
$$

for some constants $h, k>0$. It is easy to see the following topological inclusions:

$$
\mathcal{F}\left(\mathbb{R}^{n}\right) \hookrightarrow \mathcal{S}\left(\mathbb{R}^{n}\right), \quad \mathcal{S}^{\prime}\left(\mathbb{R}^{n}\right) \hookrightarrow \mathcal{F}^{\prime}\left(\mathbb{R}^{n}\right)
$$

From the above inclusions, it suffices to say that one considers (1.2) and (1.3) in the space $\mathcal{F}^{\prime}\left(\mathbb{R}^{n}\right)$.

In order to obtain the general solution and prove the Hyers-Ulam stability of (1.1) in the space $\mathcal{F}^{\prime}\left(\mathbb{R}^{n}\right)$, one employs the $n$-dimensional heat kernel, that is, the fundamental solution of the heat operator $\partial_{t}-\Delta_{x}$ in $\mathbb{R}_{x}^{n} \times \mathbb{R}_{t}^{+}$given by

$$
E_{t}(x)= \begin{cases}(4 \pi t)^{-n / 2} \exp \left(-\frac{|x|^{2}}{4 t}\right), & t>0 \\ 0, & t \leq 0\end{cases}
$$


In view of $(2.1)$, one sees that $E_{t}(\cdot)$ belongs to $\mathcal{S}\left(\mathbb{R}^{n}\right)$ for each $t>0$. Thus, its Gauss transform

$$
\tilde{u}(x, t)=\left(u * E_{t}\right)(x)=\left\langle u_{y}, E_{t}(x-y)\right\rangle, \quad x \in \mathbb{R}^{n}, t>0,
$$

is well defined for each $u \in \mathcal{F}^{\prime}\left(\mathbb{R}^{n}\right)$. In relation to the Gauss transform, it is well known that the semigroup property of the heat kernel

$$
\left(E_{t} * E_{s}\right)(x)=E_{t+s}(x)
$$

holds for convolution. Moreover, the following result holds [20].

Let $u \in \mathcal{S}^{\prime}\left(\mathbb{R}^{n}\right)$. Then, its Gauss transform $\tilde{u}(x, t)$ is a $C^{\infty}$-solution of the heat equation

$$
\left(\frac{\partial}{\partial t}-\Delta\right) \tilde{u}(x, t)=0
$$

satisfying what follows.

(i) There exist positive constants $C, M$, and $N$ such that

$$
|\tilde{u}(x, t)| \leq C t^{-M}(1+|x|)^{N} \text { in } \mathbb{R}^{n} \times(0, \delta) .
$$

(ii) $\tilde{u}(x, t) \rightarrow u$ as $t \rightarrow 0^{+}$in the sense that for every $\varphi \in \mathcal{S}\left(\mathbb{R}^{n}\right)$,

$$
\langle u, \varphi\rangle=\lim _{t \rightarrow 0^{+}} \int \tilde{u}(x, t) \varphi(x) d x .
$$

Conversely, every $C^{\infty}$-solution $U(x, t)$ of the heat equation satisfying the growth condition (2.10) can be uniquely expressed as $U(x, t)=\tilde{u}(x, t)$ for some $u \in \mathcal{S}^{\prime}\left(\mathbb{R}^{n}\right)$.

Analogously, we can represent Fourier hyperfunctions as initial values of solutions of the heat equation as a special case of the results [21]. In this case, the estimate (2.10) is replaced by what follows.

For every $\epsilon>0$, there exists a positive constant $C_{\epsilon}$ such that

$$
|\tilde{u}(x, t)| \leq C_{\epsilon} \exp \left(\epsilon\left(|x|+\frac{1}{t}\right)\right) \text { in } \mathbb{R}^{n} \times(0, \delta) .
$$




\section{General solution and stability in $\mathcal{F}^{\prime}\left(\mathbb{R}^{n}\right)$}

We will now consider the general solution and the Hyers-Ulam stability of (1.1) in the space $F^{\prime}\left(\mathbb{R}^{n}\right)$. Convolving the tensor product $E_{t}(\xi) E_{s}(\eta) E_{r}(\zeta)$ of $n$-dimensional heat kernels in both sides of (1.2), we have

$$
\begin{aligned}
{\left[(u \circ A) *\left(E_{t}(\xi) E_{s}(\eta) E_{r}(\zeta)\right)\right](x, y, z) } & =\left\langle u \circ A, E_{t}(x-\xi) E_{s}(y-\eta) E_{r}(z-\zeta)\right\rangle \\
& =\left\langle u_{\xi}, \iint E_{t}(x-\xi+\eta+\zeta) E_{s}(y-\eta) E_{r}(z-\zeta) d \eta d \zeta\right\rangle \\
& =\left\langle u_{\xi} \iint E_{t}(x+y+z-\xi-\eta-\zeta) E_{s}(\eta) E_{r}(\zeta) d \eta d \zeta\right\rangle \\
& =\left\langle u_{\xi^{\prime}}\left(E_{t} * E_{s} * E_{r}\right)(x+y+z-\xi)\right\rangle \\
& =\left\langle u_{\xi}\left(E_{t+s+r}\right)(x+y+z-\xi)\right\rangle \\
& =\tilde{u}(x+y+z, t+s+r),
\end{aligned}
$$

and similarly we obtain

$$
\begin{aligned}
& {\left[\left(u \circ P_{1}\right) *\left(E_{t}(\xi) E_{s}(\eta) E_{r}(\zeta)\right)\right](x, y, z)=\tilde{u}(x, t),} \\
& {\left[\left(u \circ P_{2}\right) *\left(E_{t}(\xi) E_{s}(\eta) E_{r}(\zeta)\right)\right](x, y, z)=\tilde{u}(y, s),} \\
& {\left[\left(u \circ P_{3}\right) *\left(E_{t}(\xi) E_{s}(\eta) E_{r}(\zeta)\right)\right](x, y, z)=\tilde{u}(z, r),} \\
& {\left[\left(u \circ B_{1}\right) *\left(E_{t}(\xi) E_{s}(\eta) E_{r}(\zeta)\right)\right](x, y, z)=\tilde{u}(x+y, t+s),} \\
& {\left[\left(u \circ B_{2}\right) *\left(E_{t}(\xi) E_{s}(\eta) E_{r}(\zeta)\right)\right](x, y, z)=\tilde{u}(y+z, s+r),} \\
& {\left[\left(u \circ B_{3}\right) *\left(E_{t}(\xi) E_{s}(\eta) E_{r}(\zeta)\right)\right](x, y, z)=\tilde{u}(z+x, r+t),}
\end{aligned}
$$

where $\tilde{u}$ is the Gauss transform of $u$. Thus, (1.2) is converted into the classical functional equation

$$
\widetilde{u}(x+y+z, t+s+r)+\widetilde{u}(x, t)+\widetilde{u}(y, s)+\widetilde{u}(z, r)=\widetilde{u}(x+y, t+s)+\widetilde{u}(y+z, s+r)+\widetilde{u}(z+x, r+t)
$$

for all $x, y, z \in \mathbb{R}^{n}$ and $t, s, r>0$. For that reason, we first prove the following lemma which is essential to prove the main result.

Lemma 3.1. Suppose that a function $f: \mathbb{R}^{n} \times(0, \infty) \rightarrow \mathbb{C}$ satisfies

$f(x+y+z, t+s+r)+f(x, t)+f(y, s)+f(z, r)=f(x+y, t+s)+f(y+z, s+r)+f(z+x, r+t)$ 
for all $x, y, z \in \mathbb{R}^{n}$ and $t, s, r>0$. Also, assume that $f(x, t)$ is continuous and 2-times differentiable with respect to $x$ and $t$, respectively. Then, there exist constants $a_{i j}, b_{i}, c_{i}, d, e \in \mathbb{C}$ such that

$$
f(x, t)=\sum_{1 \leq i \leq j \leq n} a_{i j} x_{i} x_{j}+\sum_{1 \leq i \leq n} b_{i} x_{i}+t \sum_{1 \leq i \leq n} c_{i} x_{i}+d t^{2}+e t
$$

for all $x=\left(x_{1}, \ldots, x_{n}\right) \in \mathbb{R}^{n}$ and $t>0$.

Proof. In view of (3.4), $f\left(x, 0^{+}\right):=\lim _{t \rightarrow 0^{+}} f(x, t)$ exists for each $x \in \mathbb{R}^{n}$. Letting $t=s=r \rightarrow 0^{+}$ in (3.4), we see that $f\left(x, 0^{+}\right)$satisfies (1.1). By the result as that in [15], there exist a symmetric biadditive function $B$ and an additive function $A$ such that

$$
f\left(x, 0^{+}\right)=B(x, x)+A(x)
$$

for all $x \in \mathbb{R}^{n}$. From the hypothesis that $f(x, t)$ is continuous with respect to $x$, we have

$$
f\left(x, 0^{+}\right)=\sum_{1 \leq i \leq j \leq n} a_{i j} x_{i} x_{j}+\sum_{1 \leq i \leq n} b_{i} x_{i}
$$

for some $a_{i j}, b_{i} \in \mathbb{C}$. We now define a function $h$ as $h(x, t):=f(x, t)-f\left(x, 0^{+}\right)-f(0, t)$ for all $x \in \mathbb{R}^{n}$ and $t>0$. Putting $x=y=z=0$ and $t=s=r \rightarrow 0^{+}$in (3.4), we have $f\left(0,0^{+}\right)=0$. From the definition of $h$ and $f\left(0,0^{+}\right)=0$, we see that $h$ satisfies $h(0, t)=0, h\left(x, 0^{+}\right)=0$, and

$h(x+y+z, t+s+r)+h(x, t)+h(y, s)+h(z, r)=h(x+y, t+s)+h(y+z, s+r)+h(z+x, r+t)$

for all $x, y, z \in \mathbb{R}^{n}$ and $t, s, r>0$. Putting $y=z=0$ in (3.8), we get

$$
h(x, t+s+r)+h(x, t)=h(x, t+s)+h(x, r+t) .
$$

Now letting $t \rightarrow 0^{+}$in (3.9) yields

$$
h(x, s+r)=h(x, s)+h(x, r) .
$$

Given the continuity, $h(x, t)$ can be written as

$$
h(x, t)=h(x, 1) t
$$

for all $x \in \mathbb{R}^{n}$ and $t>0$. Setting $x=0, t=1$, and $s=r \rightarrow 0^{+}$in (3.8), we obtain

$$
h(y+z, 1)=h(y, 1)+h(z, 1)
$$

for all $y, z \in \mathbb{R}^{n}$. This shows that $h(x, 1)$ is additive. Thus, $h(x, t)$ can be written in the form

$$
h(x, t)=t \sum_{1 \leq i \leq n} c_{i} x_{i}
$$


for some $c_{i} \in \mathbb{C}$. Now we are going to find the general solution of $f(0, t)$. Putting $x=y=z=0$ in (3.4), we obtain

$$
f(0, t+s+r)+f(0, t)+f(0, s)+f(0, r)=f(0, t+s)+f(0, s+r)+f(0, r+t) .
$$

Differentiating (3.14) with respect to $t$, we have

$$
f^{\prime}(0, t+s+r)+f^{\prime}(0, t)=f^{\prime}(0, t+s)+f^{\prime}(0, r+t)
$$

for all $t, s, r>0$. Similarly, differentiation of (3.15) with respect to $s$ yields

$$
f^{\prime \prime}(0, t+s+r)=f^{\prime \prime}(0, t+s)
$$

which shows that $f^{\prime \prime}(0, t)$ is a constant function. By virtue of $f\left(0,0^{+}\right)=0, f(0, t)$ can be written as

$$
f(0, t)=d t^{2}+e t
$$

for some $d, e \in \mathbb{C}$. Combining (3.7), (3.13), and (3.17), $f(x, t)$ can be written in the form

$$
f(x, t)=f\left(x, 0^{+}\right)+h(x, t)+f(0, t)=\sum_{1 \leq i \leq j \leq n} a_{i j} x_{i} x_{j}+\sum_{1 \leq i \leq n} b_{i} x_{i}+t \sum_{1 \leq i \leq n} c_{i} x_{i}+d t^{2}+e t
$$

for some $a_{i j}, b_{i}, c_{i}, d, e \in \mathbb{C}$. This completes the proof.

As an immediate consequence of Lemma 3.1, we establish the general solution of (1.1) in the space $\mathcal{F}^{\prime}\left(\mathbb{R}^{n}\right)$.

Theorem 3.2. Every solution $u$ in $\mathcal{F}^{\prime}\left(\mathbb{R}^{n}\right)$ of

$$
u \circ A+u \circ P_{1}+u \circ P_{2}+u \circ P_{3}=u \circ B_{1}+u \circ B_{2}+u \circ B_{3}
$$

has the form

$$
u(x)=u\left(x_{1}, \ldots, x_{n}\right)=\sum_{1 \leq i \leq j \leq n} a_{i j} x_{i} x_{j}+\sum_{1 \leq i \leq n} b_{i} x_{i}
$$

for some $a_{i j}, b_{i} \in \mathbb{C}$.

Proof. As we see above, if we convolve the tensor product $E_{t}(\xi) E_{s}(\eta) E_{r}(\zeta)$ of $n$-dimensional heat kernels in both sides of (3.19), then (3.19) is converted into the classical functional equation

$$
\tilde{u}(x+y+z, t+s+r)+\tilde{u}(x, t)+\tilde{u}(y, s)+\tilde{u}(z, r)=\tilde{u}(x+y, t+s)+\tilde{u}(y+z, s+r)+\tilde{u}(z+x, r+t)
$$


for all $x, y, z \in \mathbb{R}^{n}$ and $t, s, r>0$, where $\tilde{u}$ is the Gauss transform of $u$. According to Lemma 3.1, $\tilde{u}(x, t)$ is of the form

$$
\tilde{u}(x, t)=\sum_{1 \leq i \leq j \leq n} a_{i j} x_{i} x_{j}+\sum_{1 \leq i \leq n} b_{i} x_{i}+t \sum_{1 \leq i \leq n} c_{i} x_{i}+d t^{2}+e t
$$

for some constants $a_{i j}, b_{i}, c_{i}, d, e \in \mathbb{C}$. Now letting $t \rightarrow 0^{+}$, we have

$$
u=\sum_{1 \leq i \leq j \leq n} a_{i j} x_{i} x_{j}+\sum_{1 \leq i \leq n} b_{i} x_{i}
$$

which completes the proof.

We now in a position to state and prove the main result of this paper.

Theorem 3.3. Suppose that $u$ in $\mathcal{F}^{\prime}\left(\mathbb{R}^{n}\right)$ satisfies the inequality

$$
\left\|u \circ A+u \circ P_{1}+u \circ P_{2}+u \circ P_{3}-u \circ B_{1}-u \circ B_{2}-u \circ B_{3}\right\| \leq \varepsilon .
$$

Then, there exists a function $T$ defined by

$$
T(x)=\sum_{1 \leq i \leq j \leq n} a_{i j} x_{i} x_{j}+\sum_{1 \leq i \leq n} b_{i} x_{i}, \quad a_{i j}, b_{i} \in \mathbb{C},
$$

such that

$$
\|u-T(x)\| \leq \frac{13}{3} \varepsilon
$$

Proof. Convolving the tensor product $E_{t}(\xi) E_{s}(\eta) E_{r}(\zeta)$ of $n$-dimensional heat kernels in both sides of (3.24), we have the classical functional inequality

$$
|\tilde{u}(x+y+z, t+s+r)+\tilde{u}(x, t)+\tilde{u}(y, s)+\tilde{u}(z, r)-\tilde{u}(x+y, t+s)-\tilde{u}(y+z, s+r)-\tilde{u}(z+x, r+t)| \leq \epsilon
$$

for all $x, y, z \in \mathbb{R}^{n}$ and $t, s, r>0$, where $\tilde{u}$ is the Gauss transform of $u$. Define a function $f_{e}: \mathbb{R}^{n} \times(0, \infty) \rightarrow \mathbb{C}$ by $f_{e}(x, t):=(1 / 2)(\tilde{u}(x, t)+\widetilde{u}(-x, t))-\tilde{u}(0, t)$ for all $x \in \mathbb{R}^{n}$ and $t>0$. Then, $f_{e}(-x, t)=f_{e}(x, t), f_{e}(0, t)=0$, and

$\left|f_{e}(x+y+z, t+s+r)+f_{e}(x, t)+f_{e}(y, s)+f_{e}(z, r)-f_{e}(x+y, t+s)-f_{e}(y+z, s+r)-f_{e}(z+x, r+t)\right| \leq 2 \epsilon$

for all $x, y, z \in \mathbb{R}^{n}$ and $t, s, r>0$. Replacing $z$ by $-y$ in (3.28), we have

$$
\left|f_{e}(x, t+s+r)+f_{e}(x, t)+f_{e}(y, s)+f_{e}(y, r)-f_{e}(x+y, t+s)-f_{e}(x-y, r+t)\right| \leq 2 \epsilon .
$$


Putting $y=z=0$ in (3.28) yields

$$
\left|f_{e}(x, t+s+r)+f_{e}(x, t)-f_{e}(x, t+s)-f_{e}(x, r+t)\right| \leq 2 \epsilon .
$$

Taking (3.29) into (3.30), we obtain

$$
\left|f_{e}(x+y, t+s)+f_{e}(x-y, r+t) f_{e}(x, t+s)-f_{e}(x, r+t)-f_{e}(y, s)-f_{e}(y, r)\right| \leq 4 \epsilon .
$$

Letting $t \rightarrow 0^{+}$and switching $r$ by $s$, we have

$$
\left|f_{e}(x+y, s)+f_{e}(x-y, s)-2 f_{e}(x, s)-2 f_{e}(y, s)\right| \leq 4 \epsilon
$$

Substituting $y, s$ by $x, t$, respectively, and then dividing by 4 , we lead to

$$
\left|\frac{f_{e}(2 x, t)}{4}-f_{e}(x, t)\right| \leq \epsilon .
$$

Making use of an induction argument, we obtain

$$
\left|4^{-k} f_{e}\left(2^{k} x, t\right)-f_{e}(x, t)\right| \leq \frac{4}{3} \epsilon
$$

for all $k \in \mathbb{N}, x \in \mathbb{R}^{n}$, and $t>0$. Exchanging $x$ by $2^{l} x$ in (3.34) and then dividing the result by $4^{l}$, we can see that $\left\{4^{-k} f_{e}\left(2^{k} x, t\right)\right\}$ is a Cauchy sequence which converges uniformly. Let $g(x, t)=\lim _{k \rightarrow \infty} 4^{-k} f_{e}\left(2^{k} x, t\right)$ for all $x \in \mathbb{R}^{n}$ and $t>0$. It follows from (3.28) and (3.34) that $g(x, t)$ is the unique function satisfying

$$
\begin{gathered}
g(x+y+z, t+s+r)+g(x, t)+g(y, s)+g(z, r)=g(x+y, t+s)+g(y+z, s+r)+g(z+x, r+t), \\
\left|f_{e}(x, t)-g(x, t)\right| \leq \frac{4}{3} \epsilon
\end{gathered}
$$

for all $x, y, z \in \mathbb{R}^{n}$ and $t, s, r>0$. By virtue of Lemma 3.1, $g$ is of the form

$$
g(x, t)=\sum_{1 \leq i \leq j \leq n} a_{i j} x_{i} x_{j}+\sum_{1 \leq i \leq n} b_{i} x_{i}+t \sum_{1 \leq i \leq n} c_{i} x_{i}+d t^{2}+e t
$$

for some constants $a_{i j}, b_{i}, c_{i}, d, e \in \mathbb{C}$. Since $f_{e}(-x, t)=f_{e}(x, t)$ and $f_{e}(0, t)=0$ for all $x \in \mathbb{R}^{n}$ and $t>0$, we have

$$
g(x, t)=\sum_{1 \leq i \leq j \leq n} a_{i j} x_{i} x_{j}
$$


On the other hand, let $f_{o}: \mathbb{R}^{n} \times(0, \infty) \rightarrow \mathbb{C}$ be the function defined by $f_{o}(x, t):=(1 / 2)(\tilde{u}(x, t)-$ $\tilde{u}(-x, t))$ for all $x \in \mathbb{R}^{n}$ and $t>0$. Then, $f_{o}(-x, t)=-f_{o}(x, t), f_{o}(0, t)=0$, and

$$
\left|f_{o}(x+y+z, t+s+r)+f_{o}(x, t)+f_{o}(y, s)+f_{o}(z, r)-f_{o}(x+y, t+s)-f_{o}(y+z, s+r)-f_{o}(z+x, r+t)\right| \leq \epsilon
$$

for all $x, y, z \in \mathbb{R}^{n}$ and $t, s, r>0$. Replacing $z$ by $-y$ in (3.38), we have

$$
\left|f_{o}(x, t+s+r)+f_{o}(x, t)+f_{o}(y, s)-f_{o}(y, r)-f_{o}(x+y, t+s)-f_{o}(x-y, r+t)\right| \leq \epsilon .
$$

Setting $y=z=0$ in (3.38) yields

$$
\left|f_{o}(x, t+s+r)+f_{o}(x, t)-f_{o}(x, t+s)-f_{o}(x, r+t)\right| \leq \epsilon .
$$

Adding (3.39) to (3.40), we obtain

$$
\left|f_{o}(x+y, t+s)+f_{o}(x-y, r+t)-f_{o}(x, t+s)-f_{o}(x, r+t)-f_{o}(y, s)+f_{o}(y, r)\right| \leq 2 \epsilon .
$$

Letting $t \rightarrow 0^{+}$and replacing $r$ by $s$, we have

$$
\left|f_{o}(x+y, s)+f_{o}(x-y, s)-2 f_{o}(x, s)\right| \leq 2 \epsilon .
$$

Substituting $y$, s by $x, t$, respectively, and then dividing by 2 , we lead to

$$
\left|\frac{f_{o}(2 x, t)}{2}-f_{o}(x, t)\right| \leq \epsilon
$$

Using the iterative method, we obtain

$$
\left|2^{-k} f_{o}\left(2^{k} x, t\right)-f_{o}(x, t)\right| \leq 2 \epsilon
$$

for all $k \in \mathbb{N}, x \in \mathbb{R}^{n}$, and $t>0$. From (3.38) and (3.44), we verify that $h$ is the unique function satisfying

$$
\begin{gathered}
h(x+y+z, t+s+r)+h(x, t)+h(y, s)+h(z, r)=h(x+y, t+s)+h(y+z, s+r)+h(z+x, r+t), \\
\left|f_{o}(x, t)-h(x, t)\right| \leq 2 \epsilon
\end{gathered}
$$

for all $x, y, z \in \mathbb{R}^{n}$ and $t, s, r>0$. According to Lemma 3.1, there exist $a_{i j}, b_{i}, c_{i}, d, e \in \mathbb{C}$ such that

$$
h(x, t)=\sum_{1 \leq i \leq j \leq n} a_{i j} x_{i} x_{j}+\sum_{1 \leq i \leq n} b_{i} x_{i}+t \sum_{1 \leq i \leq n} c_{i} x_{i}+d t^{2}+e t .
$$


On account of $f_{o}(-x, t)=f_{o}(x, t)$ and $f_{o}(0, t)=0$ for all $x \in \mathbb{R}^{n}$ and $t>0$, we have

$$
h(x, t)=\sum_{1 \leq i \leq n} b_{i} x_{i}+t \sum_{1 \leq i \leq n} c_{i} x_{i} .
$$

In turn, since $\tilde{u}(x, t)=f_{e}(x, t)+f_{o}(x, t)+\tilde{u}(0, t)$, we figure out

$$
\begin{aligned}
|\widetilde{u}(x, t)-g(x, t)-h(x, t)| & \leq\left|f_{e}(x, t)-g(x, t)\right|+\left|f_{o}(x, t)-h(x, t)\right|+|\widetilde{u}(0, t)| \\
& \leq \frac{10}{3} \epsilon+|\widetilde{u}(0, t)|
\end{aligned}
$$

In view of (3.27), it is easy to see that $c:=\limsup _{t \rightarrow 0^{+}} f(0, t)$ exists. Letting $x=y=z=0$ and $t=s=r \rightarrow 0^{+}$in (3.27), we have $|c| \leq \epsilon$. Finally, taking $t \rightarrow 0^{+}$in (3.48), we have

$$
\left\|u-\left(\sum_{1 \leq i \leq j \leq n} a_{i j} x_{i} x_{j}+\sum_{1 \leq i \leq n} b_{i} x_{i}\right)\right\| \leq \frac{13}{3} \epsilon
$$

which completes the proof.

Remark 3.4. The above norm inequality (3.49) implies that $u-T(x)$ belongs to $\left(L^{1}\right)^{\prime}=L^{\infty}$. Thus, all the solution $u$ in $\mathcal{F}^{\prime}\left(\mathbb{R}^{n}\right)$ can be written uniquely in the form

$$
u=T(x)+\mu,
$$

where $\mu$ is a bounded measurable function such that $\|\mu\|_{L^{\infty}} \leq(13 / 3) \epsilon$.

\section{Acknowledgment}

This work was supported by the second stage of Brain Korea 21 project, the Development Project of Human Resources in Mathematics, KAIST, 2008.

\section{References}

[1] E. Y. Deeba and E. L. Koh, "d'Alembert functional equations in distributions," Proceedings of the American Mathematical Society, vol. 116, no. 1, pp. 157-164, 1992.

[2] I. Fenyö, "On the general solution of a functional equation in the domain of distributions," Aequationes Mathematicae, vol. 3, pp. 236-246, 1969.

[3] E. L. Koh, "The Cauchy functional equations in distributions," Proceedings of the American Mathematical Society, vol. 106, no. 3, pp. 641-646, 1989.

[4] M. Neagu, "About the Pompeiu equation in distributions," Institutul Politehnic "Traian Vuia" Timişoara. Lucrările Seminarului de Matematică şi Fizică, no. 1, pp. 62-66, 1984.

[5] J. Chung and S. Lee, "Some functional equations in the spaces of generalized functions," Aequationes Mathematicae, vol. 65, no. 3, pp. 267-279, 2003.

[6] S.-Y. Chung, "Reformulation of some functional equations in the space of Gevrey distributions and regularity of solutions," Aequationes Mathematicae, vol. 59, no. 1-2, pp. 108-123, 2000.

[7] D. H. Hyers, "On the stability of the linear functional equation," Proceedings of the National Academy of Sciences of the United States of America, vol. 27, no. 4, pp. 222-224, 1941.

[8] D. H. Hyers, G. Isac, and Th. M. Rassias, Stability of Functional Equations in Several Variables, Progress in Nonlinear Differential Equations and Their Applications, 34, Birkhäuser, Boston, Mass, USA, 1998. 
[9] K.-W. Jun, Y.-H. Lee, and J. Lee, "On the stability of a new Pexider-type functional equation," Journal of Inequalities and Applications, vol. 2008, Article ID 816963, 22 pages, 2008.

[10] S. M. Ulam, Problems in Modern Mathematics, John Wiley \& Sons, New York, NY, USA, 1964.

[11] J. Chung, "Stability of functional equations in the spaces of distributions and hyperfunctions," Journal of Mathematical Analysis and Applications, vol. 286, no. 1, pp. 177-186, 2003.

[12] J. Chung, S.-Y. Chung, and D. Kim, "The stability of Cauchy equations in the space of Schwartz distributions," Journal of Mathematical Analysis and Applications, vol. 295, no. 1, pp. 107-114, 2004.

[13] J. Chung, "A distributional version of functional equations and their stabilities," Nonlinear Analysis: Theory, Methods \& Applications, vol. 62, no. 6, pp. 1037-1051, 2005.

[14] Y.-S. Lee and S.-Y. Chung, "Stability of cubic functional equation in the spaces of generalized functions," Journal of Inequalities and Applications, vol. 2007, Article ID 79893, 13 pages, 2007.

[15] Pl. Kannappan, "Quadratic functional equation and inner product spaces," Results in Mathematics, vol. 27, no. 3-4, pp. 368-372, 1995.

[16] S.-M. Jung, "On the Hyers-Ulam stability of the functional equations that have the quadratic property," Journal of Mathematical Analysis and Applications, vol. 222, no. 1, pp. 126-137, 1998.

[17] L. Hörmander, The Analysis of Linear Partial Differential Operators. I. Distribution Theory and Fourier Analysis, vol. 256 of Fundamental Principles of Mathematical Sciences, Springer, Berlin, Germany, 1983.

[18] L. Schwartz, Théorie des Distributions, Publications de l'Institut de Mathématique de l'Université de Strasbourg, no. IX-X, Hermann, Paris, France, 1966.

[19] J. Chung, S.-Y. Chung, and D. Kim, "A characterization for Fourier hyperfunctions," Publications of the Research Institute for Mathematical Sciences, vol. 30, no. 2, pp. 203-208, 1994.

[20] T. Matsuzawa, "A calculus approach to hyperfunctions. III," Nagoya Mathematical Journal, vol. 118, pp. 133-153, 1990.

[21] K. W. Kim, S.-Y. Chung, and D. Kim, "Fourier hyperfunctions as the boundary values of smooth solutions of heat equations," Publications of the Research Institute for Mathematical Sciences, vol. 29, no. 2, pp. 289-300, 1993. 